\title{
Some generalizations of common fixed point problems with applications
}

Zhe Yang*

\section{"Correspondence:}

zheyang211@163.com

School of Economics, Shanghai

University of Finance and

Economics, Shanghai, 200433,

China

Key Laboratory of Mathematical Economics (SUFE), Ministry of

Education, Shanghai, 200433, China

\begin{abstract}
In this paper, we establish a unified approach to study the existence of fixed points for common fixed point problems. Moreover, we introduce mixed systems of common fixed point problems, systems of common fixed point problems and common fixed point problems without convex assumptions. As applications, we establish a general type of some set-valued variational inequalities, and we obtain some existence theorems of solutions of set-valued variational inequalities. The results of this paper improve and generalize several known results on common fixed point problems.
\end{abstract} MSC: 47H10; 49J53; 49J40; 49J45

Keywords: common fixed point; general types; set-valued variational inequality

\section{Introduction}

In three recent papers [1-3], by using some new concepts of generalized KKM mappings, the authors established common fixed point theorems for families of set-valued mappings in Hausdorff topological vector spaces. Recently, Agarwal et al. [4] established a common fixed point theorem for a family of self set-valued mappings on a compact and convex set in a locally convex topological vector space. As applications, an existence theorem of solutions for a variational inequality of Stampacchia type and some Ky Fan-type minimax inequalities were obtained.

It is well known that the equilibrium problems are unified models of several problems, namely, optimization problems, saddle point problems, variational inequalities, fixed point problems, Nash equilibrium problems etc. Recently, Luc [5] introduced a more general model of equilibrium problems, which is called a variational relation problem (in short, VR). The stability of the solution set of variational relation problems was studied in $[6,7]$. Some various types of variational relation problems or systems of variational relation problems have been investigated in many recent papers (see [8-12]). Recently, Agarwal et al. [13] presented a unified approach in studying the existence of solutions for two types of variational relation problems, and Balaj and Lin [14] established existence criteria for the solutions of two very general types of variational relation problems (see also [15-22] for further studies of variational relation problems).

Motivated and inspired by research works mentioned above, in this paper, we establish a unified approach to study the existence of fixed points for common fixed point problems. As generalizations, mixed systems of common fixed point problems, systems of common

(O2014 Yang; licensee Springer. This is an Open Access article distributed under the terms of the Creative Commons Attribution License (http://creativecommons.org/licenses/by/2.0), which permits unrestricted use, distribution, and reproduction in any medium, provided the original work is properly cited. 
fixed point problems, and common fixed point problems without convex assumptions are obtained.

\section{Preliminary}

In this short section, we recall some definitions and known results concerning set-valued mappings which will be needed throughout this paper.

Let $X, Y, Z$ be three Hausdorff topological spaces. We adopt the following notations: for a set $U$ and point $x, \alpha_{1}(x, U)$ and, respectively, $\alpha_{2}(x, U)$ means $\forall x \in U$, and, respectively, $\exists x \in U$. Denote $\widehat{\alpha}$ by $\widehat{\alpha}_{1}=\alpha_{2}$ and $\widehat{\alpha}_{2}=\alpha_{1}$; for two sets $A, B, \gamma_{1}(A, B)$ and, respectively, $\gamma_{2}(A, B)$ means $A \subset B$, and, respectively, $A \cap B \neq \emptyset$. Denote $\widehat{\gamma}$ by $\widehat{\gamma_{1}}=\gamma_{2}$ and $\widehat{\gamma_{2}}=\gamma_{1}$. A setvalued mapping $F: X \rightrightarrows Y$ is said to be: (1) upper semicontinuous at $x \in X$ if, for any open subset $O$ of $Y$ with $O \supset F(x)$, there exists an open neighborhood $U(x)$ of $x$ such that $O \supset F\left(x^{\prime}\right)$ for any $x^{\prime} \in U(x)$; (2) upper semicontinuous on $X$, if it is upper semicontinuous at each $x \in X$; (3) lower semicontinuous at $x \in X$ if, for any open subset $O$ of $Y$ with $O \cap F(x) \neq$ $\emptyset$, there exists an open neighborhood $U(x)$ of $x$ such that $O \cap F\left(x^{\prime}\right) \neq \emptyset$ for any $x^{\prime} \in U(x)$; (4 lower semicontinuous on $X$, if it is lower semicontinuous in each $x \in X$; (5) closed if $\operatorname{Graph}(F)=\{(x, y) \in X \times Y \mid y \in F(x)\}$ is a closed subset of $X \times Y$.

Lemma 2.1 (Corollary 17.55 (Kakutani-Fan-Glicksberg fixed point theorem) of [23]) Let $X$ be a nonempty, convex and compact subset of a locally convex topological linear space, and $F: X \rightrightarrows X$ be an upper semicontinuous set-valued mapping with nonempty convex compact values. Then there exists $x^{*} \in X$ such that $x^{*} \in F\left(x^{*}\right)$.

Lemma 2.2 (Lemma 17.8, Theorem 17.10, Theorem 17.16, Theorem 17.19 of [23])

(i) The image of a compact set under a compact-valued upper semicontinuous set-valued mapping is compact.

(ii) If an upper semicontinuous set-valued mapping possess compact-valued, then it is closed.

(iii) The correspondence $\varphi$ is upper semicontinuous at $x$ and $\varphi(x)$ is compact, if and only if, for every net $\left\{\left(x^{\alpha}, y^{\alpha}\right)\right\}$ in the graph of $\varphi$, that is, with $y^{\alpha} \in \varphi\left(x^{\alpha}\right)$ for each $\alpha$, if $x^{\alpha} \longrightarrow x$, then the net $\left\{y^{\alpha}\right\}$ has a limit point in $\varphi(x)$.

(iv) If $X$ and $Y$ are topological spaces, a set-valued mapping $F: X \rightrightarrows Y$ is lower semicontinuous, if and only if, for any net $\left\{x_{\alpha}\right\}$ in $X$, converging to $x \in X$, and each $y \in F(x)$, there exists a net $\left\{y_{\alpha}\right\}$ converging to $y$, with $y_{\alpha} \in F\left(x_{\alpha}\right)$ for all $\alpha$.

\section{Main results}

Let $S: X \rightrightarrows X, Q: X \rightrightarrows Y, T: Y \times Z \rightrightarrows Z$ and $P: X \rightrightarrows Z$ be set-valued mappings with nonempty values. A common fixed point problem of type $\alpha$ (CFP- $\alpha$ ) consists in finding $\tilde{x} \in X$ such that $\tilde{x} \in S(\tilde{x})$, for any $y \in Q(\tilde{x})$,

$$
\alpha(z, P(\tilde{x})), \quad z \in T(y, z) .
$$

When $\alpha=\alpha_{1}$, a common fixed point problem of type $\alpha_{1}$ (CFP- $\left.\alpha_{1}\right)$ consists in finding $\tilde{x} \in X$ such that $\tilde{x} \in S(\tilde{x})$, and

$$
z \in \bigcap_{y \in Q(\tilde{x})} T(y, z), \quad \forall z \in P(\tilde{x})
$$


When $\alpha=\alpha_{2}$, a common fixed point problem of type $\alpha_{2}\left(\right.$ CFP- $\left.\alpha_{2}\right)$ consists in finding $\tilde{x} \in X$ such that $\tilde{x} \in S(\tilde{x})$ and, for any $y \in Q(\tilde{x})$, there exists $\tilde{z} \in P(\tilde{x})$ for which

$$
\tilde{z} \in T(y, \tilde{z}) .
$$

When $X=Z$ and $P(x)=\{x\}$ for any $x \in X$, the problems (CFP- $\left.\alpha_{1}\right)$ and (CFP- $\left.\alpha_{2}\right)$ reduce the common fixed point problem (CFP): finding $\tilde{x} \in X$ such that $\tilde{x} \in S(\tilde{x})$, and

$$
\tilde{x} \in \bigcap_{y \in Q(\tilde{x})} T(y, \tilde{x}) .
$$

Theorem 3.1 Assume that the data of problem (CFP- $\alpha$ ) satisfy the following conditions:

(i) $X$ is a nonempty, convex and compact subset of a locally convex topological linear space, $Y$ is Hausdorff linear topological space, and $Z$ is a Hausdorff topological space;

(ii) $S$ is upper semicontinuous with nonempty convex compact values;

(iii) $U(y):=\{x \in X \mid y \in Q(x)$ and $\widehat{\alpha}(z, P(x)), z \notin T(y, z)\}$ is open in $X$.

Moreover, assume that there exists a set-valued mapping $F: Y \rightrightarrows X$ such that

(iv) $F(Q(x)) \subset S(x)$ for any $x \in X$;

(v) $F(y)$ is nonempty, convex and compact for any $y \in Y$;

(vi) $F$ is convex, i.e., $\sum_{j=1}^{n} \lambda_{j} F\left(y_{j}\right) \subset F\left(\sum_{j=1}^{n} \lambda_{j} y_{j}\right)$ for any $y_{j} \in Y$ and any $\lambda_{j} \geq 0$ with $\sum_{j=1}^{n} \lambda_{j}=1$;

(vii) $T$ is $\alpha-(F, P)-\mathrm{KKM}$, i.e., for any finite set $\left\{y_{1}, \ldots, y_{n}\right\}$ of $Y$ and any $x \in F\left(\operatorname{co}\left\{y_{1}, \ldots, y_{n}\right\}\right)$, there is $i_{0} \in\{1, \ldots, n\}$ such that $\alpha(z, P(x)), z \in T\left(y_{i_{0}}, z\right)$.

Then problem $(C F P-\alpha)$ has at least a solution.

Proof By way of contradiction suppose that, for any $x \in X, x \notin S(x)$, or, there is $y \in Q(x)$ such that $\widehat{\alpha}(z, P(x)), z \notin T(y, z)$. Denote $U_{0}=\{x \in X \mid x \notin S(x)\}$, which is open in $X$ by (ii). Therefore,

$$
X=U_{0} \cup \bigcup_{y \in Y} U(y)
$$

By (iii), there is a finite subset $\left\{y_{1}, \ldots, y_{n}\right\}$ of $Y$ such that

$$
X=U_{0} \cup \bigcup_{k=1}^{n} U\left(y_{k}\right)
$$

Let $\left\{\beta_{k} \mid k=0,1,2, \ldots, n\right\}$ be the partition of unity subordinate to the open covering $\left\{U_{0}, U\left(y_{k}\right) \mid k=1,2, \ldots, n\right\}$ of $X$, i.e., $\left\{\beta_{k} \mid k=0,1,2, \ldots, n\right\}$ is a set of continuous functions with following properties: $0 \leq \beta_{k}(x) \leq 1, \sum_{k=0}^{n} \beta_{k}(x)=1, \forall x \in X, k=0,1,2, \ldots, n$; and if $x \notin U\left(y_{k}\right)$, for some $k \in\{1, \ldots, n\}$, then $\beta_{k}(x)=0$, and if $x \notin U_{0}$, then $\beta_{0}(x)=0$.

Now, we define the following set-valued mapping $\phi: X \rightrightarrows X$ :

$$
\phi(x)=\beta_{0}(x) S(x)+\sum_{k=1}^{n} \beta_{k}(x) F\left(y_{k}\right)
$$


Clearly $\phi$ is upper semicontinuous on $X$. Moreover, since $S(x)$ and $F\left(y_{k}\right)$ are nonempty, convex and compact, $\phi(x)$ is nonempty, convex and compact in $X$ for any $x \in X$. By Lemma 2.1, there exists $x^{*} \in X$ such that $x^{*} \in \phi\left(x^{*}\right)$. Let $I\left(x^{*}\right)=\left\{k \in\{1, \ldots, n\} \mid \beta_{k}\left(x^{*}\right)>0\right\}$. Then, for any $k \in I\left(x^{*}\right), x^{*} \in U\left(y_{k}\right)$, it follows that $y_{k} \in Q\left(x^{*}\right)$ for any $k \in I\left(x^{*}\right)$. By (iv), $F\left(y_{k}\right) \subset F\left(Q\left(x^{*}\right)\right) \subset S\left(x^{*}\right)$ for any $k \in I\left(x^{*}\right)$. Therefore, $x^{*} \in \phi\left(x^{*}\right) \subset S\left(x^{*}\right)$, which implies that $\beta_{0}\left(x^{*}\right)=0$. It follows from the convexity of $F$ that

$$
x^{*} \in \sum_{k=1}^{n} \beta_{k}\left(x^{*}\right) F\left(y_{k}\right) \subset F\left(\sum_{k \in I\left(x^{*}\right)} \beta_{k}\left(x^{*}\right) y_{k}\right) .
$$

By (vii), there is $i_{0} \in I\left(x^{*}\right)$ such that $\alpha\left(z, P\left(x^{*}\right)\right), z \in T\left(y_{i_{0}}, z\right)$, which implies that $x^{*} \notin U\left(y_{i_{0}}\right)$, i.e., $\beta_{i_{0}}\left(x^{*}\right)=0$. It contradicts the fact that $i_{0} \in I\left(x^{*}\right)$, that is, $\beta_{i_{0}}\left(x^{*}\right)>0$. This completes the proof.

Remark 3.1 By Proposition 3.3 of [14], when $\alpha=\alpha_{1}$, condition (iii) in Theorem 3.1 can be replaced by (a1) $P: X \rightrightarrows Z$ is lower semicontinuous; (b1) $\{z \in Z \mid z \in T(y, z)\}$ is closed in $X$ for any $y \in Y$; (c1) $Q^{-1}(y)$ is open in $X$ for any $y \in Y$. Thus we have the following theorem.

Theorem 3.2 Assume that the data of problem (CFP- $\left.\alpha_{1}\right)$ satisfy the conditions (i), (ii), (iv)-(vi) of Theorem 3.1 and

(a1) $P: X \rightrightarrows Z$ is lower semicontinuous;

(b1) $\{z \in Z \mid z \in T(y, z)\}$ is closed in $X$ for any $y \in Y$;

(c1) $Q^{-1}(y)$ is open in $X$ for any $y \in Y$;

(d1) $T$ is $\alpha_{1}-(F, P)-\mathrm{KKM}$, i.e., for any finite set $\left\{y_{1}, \ldots, y_{n}\right\}$ of $Y$ and any $x \in F\left(\operatorname{co}\left\{y_{1}, \ldots, y_{n}\right\}\right)$, there is $i_{0} \in\{1, \ldots, n\}$ such that $z \in T\left(y_{i_{0}}, z\right)$ for any $z \in P(x)$.

Then problem $\left(C F P-\alpha_{1}\right)$ has at least a solution.

Remark 3.2 By Proposition 3.1 of [14], when $\alpha=\alpha_{2}$, condition (iii) in Theorem 3.1 can be replaced by (a2) $P: X \rightrightarrows Z$ is upper semicontinuous with nonempty compact values; (b2) $\{z \in Z \mid z \in T(y, z)\}$ is closed in $X$ for any $y \in Y$; (c2) $Q^{-1}(y)$ is open in $X$ for any $y \in Y$. Thus we have the following theorem.

Theorem 3.3 Assume that the data of problem $\left(C F P-\alpha_{2}\right)$ satisfy the conditions (i), (ii), (iv)-(vi) of Theorem 3.1 and

(a2) $P: X \rightrightarrows Z$ is upper semicontinuous with nonempty compact values;

(b2) $\{z \in Z \mid z \in T(y, z)\}$ is closed in $X$ for any $y \in Y$;

(c2) $Q^{-1}(y)$ is open in $X$ for any $y \in Y$;

(d2) $T$ is $\alpha_{2}-(F, P)-\mathrm{KKM}$, i.e., for any finite set $\left\{y_{1}, \ldots, y_{n}\right\}$ of $Y$ and any $x \in F\left(\operatorname{co}\left\{y_{1}, \ldots, y_{n}\right\}\right)$, there is $i_{0} \in\{1, \ldots, n\}$ such that there is $z \in P(x)$ for which $z \in T\left(y_{i_{0}}, z\right)$.

Then problem $\left(C F P-\alpha_{2}\right)$ has at least a solution.

$$
\text { If } X=Y \text { and } F(x)=x \text { for all } x \in X \text {, we have problem (CFP- } \alpha \text { ). }
$$

Theorem 3.4 Let $X$ be a nonempty, convex and compact subset of a locally convex topological linear space, $Z$ be a Hausdorff topological space, and $S, Q: X \rightrightarrows X, T: X \times Z \rightrightarrows Z$ and $P: X \rightrightarrows Z$ be set-valued mappings with nonempty values. Assume that 
(i) $S$ is upper semicontinuous with nonempty convex compact values, and $Q(x) \subset S(x)$ for all $x \in X$;

(ii) the set $\{x \in X \mid y \in Q(x)$ and $\widehat{\alpha}(z, P(x)), z \notin T(y, z)\}$ is open in $X$ for any $y \in X$;

(iii) $T$ is $\alpha$-P-KKM, i.e., for any finite set $\left\{x_{1}, \ldots, x_{n}\right\}$ of $X$ and any $x \in \operatorname{co}\left\{x_{1}, \ldots, x_{n}\right\}$, there is $i_{0} \in\{1, \ldots, n\}$ such that $\alpha(z, P(x)), z \in T\left(x_{i_{0}}, z\right)$.

Then problem (CFP- $\alpha)$ has at least a solution.

When $X=Y=Z$, and $F(x)=x, P(x)=\{x\}$ for all $x \in X$, we obtain the following corollary.

Corollary 3.1 Let $X$ be a nonempty, convex, and compact subset of a locally convex topological linear space, and $S, Q: X \rightrightarrows X, T: X \times X \rightrightarrows X$ be set-valued mappings with nonempty values. Assume that

(i) $S$ is upper semicontinuous with nonempty convex compact values;

(ii) $Q^{-1}(y)$ is open in $X$ for any $y \in X$, and $\emptyset \neq Q(x) \subset S(x)$ for any $x \in X$;

(iii) the set $\{x \in X \mid x \in T(y, x)\}$ is closed in $X$ for any $y \in X$;

(iv) for any finite set $\left\{x_{1}, \ldots, x_{n}\right\}$ of $X$ and any $x \in \operatorname{co}\left\{x_{1}, \ldots, x_{n}\right\}$, there is $i_{0} \in\{1, \ldots, n\}$ such that $x \in T\left(x_{i_{0}}, x\right)$.

Then there exists $\tilde{x} \in X$ such that $\tilde{x} \in S(\tilde{x})$ and $\tilde{x} \in \bigcap_{y \in Q(\tilde{x})} T(y, \tilde{x})$.

Remark 3.3 Theorems 3.1-3.4 generalize the results of [1-4]. When $X=Y$ and $P(x)=$ $F(x)=x$ for all $x \in X$, and $S(x)=Q(x)=X$ for any $x \in X$, our Theorems 3.1-3.4 reduce to the results of $[1-4]$.

Now, we introduce a new class of problems, called mixed systems of common fixed point problems (MSCFP). Let $X, Y$ be nonempty sets in two Hausdorff topological vector spaces, $Z$ be a Hausdorff topological space, $S: X \times Y \rightrightarrows X, H: X \times Y \rightrightarrows Y, Q: X \times Y \rightrightarrows X, M$ : $X \times Y \rightrightarrows Y, T: X \times Z \rightrightarrows Z, F: Y \times Z \rightrightarrows Z, P: X \times Y \rightrightarrows Z$ be set-valued mappings with nonempty values. A mixed system of common fixed point problems consists in finding $\left(x^{*}, y^{*}\right) \in X \times Y$ such that $x^{*} \in S\left(x^{*}, y^{*}\right), y^{*} \in H\left(x^{*}, y^{*}\right)$ and

$$
\begin{array}{lll}
\forall u \in Q\left(x^{*}, y^{*}\right), & \forall z \in P\left(x^{*}, y^{*}\right), & \text { s.t. } z \in T(u, z), \\
\forall v \in M\left(x^{*}, y^{*}\right), & \exists z \in P\left(x^{*}, y^{*}\right), & \text { s.t. } z \in F(v, z) .
\end{array}
$$

\section{Theorem 3.5 Assume that}

(i) $X, Y, Z$ are three nonempty, compact and convex subsets of three Hausdorff linear topological spaces;

(ii) $C=\{(x, y) \in X \times Y: x \in S(x, y)\}$ and $D=\{(x, y) \in X \times Y: y \in H(x, y)\}$ are nonempty and closed in $X \times Y$;

(iii) $P$ is continuous with nonempty convex compact values;

(iv) $\{z \in Z: z \in T(x, z)\}$ and $\{z \in Z: z \in F(y, z)\}$ are closed for any $(x, y) \in X \times Y$;

(v) $Q(x, y) \neq \emptyset, M(x, y) \neq \emptyset, c o Q(x, y) \subset S(x, y), \operatorname{co} M(x, y) \subset H(x, y)$, and $Q^{-1}(x), M^{-1}(y)$ are open for any $(x, y) \in X \times Y$;

(vi) for any fixed $y \in Y$, any finite subset $\left\{u_{1}, \ldots, u_{n}\right\}$ of $X$ and any $x \in \operatorname{co}\left\{u_{1}, \ldots, u_{n}\right\}$, there is $i \in\{1, \ldots, n\}$ such that, for any $z \in P(x, y), z \in T\left(u_{i}, z\right)$;

(vii) for any fixed $x \in X$, any finite subset $\left\{v_{1}, \ldots, v_{n}\right\}$ of $Y$ and any $y \in \operatorname{co}\left\{v_{1}, \ldots, v_{n}\right\}$, there is $i \in\{1, \ldots, n\}$ such that there is $z \in P(x, y)$ for which $z \in F\left(v_{i}, z\right)$.

Then problem (MSCFP) has at least a solution. 
Proof Define $A: X \times Y \rightrightarrows X$ and $B: X \times Y \rightrightarrows Y$ as follows:

$$
\begin{aligned}
& A(x, y)=\{u \in X: \exists z \in P(x, y), z \notin T(u, z)\}, \\
& B(x, y)=\{v \in Y: z \notin F(v, z), \forall z \in P(x, y)\} .
\end{aligned}
$$

By (iii), (iv), and Remarks 3.1, 3.2, $A^{-1}(u), B^{-1}(v)$ are open in $X \times Y$ for any $(u, v) \in X \times Y$.

Suppose there exists $(x, y) \in X \times Y$ such that $x \in \operatorname{coA}(x, y)$, then there is a finite subset $\left\{u_{1}, \ldots, u_{n}\right\}$ of $A(x, y)$ such that $x \in \operatorname{co}\left\{u_{1}, \ldots, u_{n}\right\}$. By (vi), there is $i_{0} \in\{1, \ldots, n\}$ such that $z \in$ $T\left(u_{i_{0}}, z\right)$ for any $z \in P(x, y)$, which contradicts the fact that $u_{i} \in A(x, y)$ for any $i \in\{1, \ldots, n\}$. Hence $x \notin \operatorname{coA}(x, y)$ for any $(x, y) \in X \times Y$.

Suppose there exists $(x, y) \in X \times Y$ such that $y \in \operatorname{coB}(x, y)$, then there is a finite subset $\left\{v_{1}, \ldots, v_{n}\right\}$ of $B(x, y)$ such that $y \in \operatorname{co}\left\{v_{1}, \ldots, v_{n}\right\}$. By (vii), there is $i_{0} \in\{1, \ldots, n\}$ such that there exists $z \in P(x, y)$ for which $z \in F\left(v_{i_{0}}, z\right)$, which contradicts the fact that $v_{i} \in B(x, y)$ for any $i \in\{1, \ldots, n\}$. Hence $y \notin \operatorname{co} B(x, y)$ for any $(x, y) \in X \times Y$.

Define the mappings $A^{\prime}: X \times Y \rightrightarrows X$ and $B^{\prime}: X \times Y \rightrightarrows Y$ as follows:

$$
\begin{aligned}
& A^{\prime}(x, y)= \begin{cases}A(x, y) \cap Q(x, y), & \text { if }(x, y) \in C, \\
Q(x, y), & \text { if }(x, y) \notin C,\end{cases} \\
& B^{\prime}(x, y)= \begin{cases}B(x, y) \cap M(x, y), & \text { if }(x, y) \in D, \\
M(x, y), & \text { if }(x, y) \notin D .\end{cases}
\end{aligned}
$$

For any $u \in X$,

$$
A^{\prime-1}(u)=\left[Q^{-1}(u) \cap A^{-1}(u)\right] \cup\left[((X \times Y) \backslash C) \cap Q^{-1}(u)\right]
$$

is open in $X \times Y$. Similarly, $B^{\prime-1}(v)$ is open for any $v \in Y$. Hence, $A^{\prime-1}(u), B^{\prime-1}(v)$ are open for any $(u, v) \in X \times Y$, and $x \notin c o A^{\prime}(x, y), y \notin c o B^{\prime}(x, y)$ for any $(x, y) \in X \times Y$. By Theorem 3 of [24], there exists $\left(x^{*}, y^{*}\right) \in X \times Y$ such that $A^{\prime}\left(x^{*}, y^{*}\right)=\emptyset$ and $B^{\prime}\left(x^{*}, y^{*}\right)=\emptyset$, which implies that $x^{*} \in S\left(x^{*}, y^{*}\right), y^{*} \in H\left(x^{*}, y^{*}\right)$ and

$$
\begin{array}{lll}
\forall u \in Q\left(x^{*}, y^{*}\right), & \forall z \in P\left(x^{*}, y^{*}\right), & \text { s.t. } z \in T(u, z), \\
\forall v \in M\left(x^{*}, y^{*}\right), & \exists z \in P\left(x^{*}, y^{*}\right), & \text { s.t. } z \in F(v, z) .
\end{array}
$$

As a generalization, we introduce the following system of common fixed point problems. Let $I$ be any index set. For any $i \in I$, let $X_{i}, Z_{i}$ be Hausdorff topological spaces, and $S_{i}: X \rightrightarrows$ $X_{i}, Q_{i}: X \rightrightarrows X_{i}, T_{i}: X_{i} \times Z_{i} \rightrightarrows Z_{i}$ and $P_{i}: X \rightrightarrows Z_{i}$ be set-valued mappings with nonempty values. A system of common fixed point problems (SCFP) consists in finding $\tilde{x} \in X$ such that, for each $i \in I, \tilde{x}_{i} \in S_{i}(\tilde{x})$ and, for any $y_{i} \in Q_{i}(\tilde{x})$,

$$
\alpha\left(z_{i}, P_{i}(\tilde{x})\right), \quad z_{i} \in T_{i}\left(y_{i}, z_{i}\right)
$$

Theorem 3.6 Assume that, for each $i \in I$, the following conditions are satisfied:

(i) $X_{i}$ is a nonempty, convex and compact subset of a locally convex topological linear space, and $Z_{i}$ is a Hausdorff topological space; 
(ii) the set $\Omega_{i}:=\left\{x \in X: x_{i} \in S(x)\right\}$ is nonempty and closed in $X$;

(iii) the set-valued mapping $G_{i}: X \rightrightarrows X_{i}$, defined by $G_{i}(x):=\left\{y_{i} \in X_{i} \mid \widehat{\alpha}\left(z_{i}, P_{i}(x)\right), z_{i} \notin T_{i}\left(y_{i}, z_{i}\right)\right\}$, has open fibers;

(iv) $Q_{i}^{-1}\left(y_{i}\right)$ is open in $X$ for any $y_{i} \in X_{i}$;

(v) for any finite set $\left\{y_{i 1}, \ldots, y_{i n}\right\}$ of $X_{i}$ and any $x_{i} \in \operatorname{co}\left\{y_{i 1}, \ldots, y_{i n}\right\}$ with $x=\left(x_{i}\right)_{i \in I}$, there is $j \in\{1, \ldots, n\}$ such that $\alpha\left(z_{i}, P_{i}(x)\right), z_{i} \in T_{i}\left(y_{i j}, z_{i}\right)$.

Then problem (SCFP) has at least a solution.

Proof For each $i \in I$, define the set

$$
W_{i}=\left[X \backslash \Omega_{i}\right] \cup\left\{x \in X \mid G_{i}(x) \cap Q_{i}(x) \neq \emptyset\right\} .
$$

By (ii) and (iii), $W_{i}$ is open in $X$ for each $i \in I$. Since $G_{i}^{-1}\left(y_{i}\right)$ is open in $X$ for each $i \in I$, by a known continuous selection Theorem (see [23, Theorem 17.63]), there is a continuous function $f_{i}: X \longrightarrow X_{i}$ such that $f_{i}(x) \in \operatorname{coG}_{i}(x)$. Thus, for each $i \in I$, we define the mapping $F: X \rightrightarrows X$ as follows:

$$
F_{i}(x)=\left\{\begin{array}{ll}
f_{i}(x), & \text { if } x \in W_{i}, \\
X_{i}, & \text { if } x \notin W_{i},
\end{array} \quad F(x)=\prod_{i \in I} F_{i}(x) .\right.
$$

By Lemma 2.1, there exists $x^{*} \in X$ such that $x^{*} \in F\left(x^{*}\right)$, which implies that $x_{i}^{*} \in F_{i}\left(x^{*}\right)$ for each $i \in I$. If $x \in W_{i_{0}}$ for some $i_{0} \in I, x_{i_{0}}^{*}=f_{i_{0}}\left(x^{*}\right) \in \operatorname{coG}_{i_{0}}\left(x^{*}\right)$. Then there exists a finite set $\left\{y_{i_{0}}, \ldots, y_{i_{0} n}\right\} \subset G_{i_{0}}\left(x^{*}\right)$ such that $x_{i_{0}}^{*} \in \operatorname{co}\left\{y_{i_{0}}, \ldots, y_{i_{0} n}\right\}$. By (v), there is $j_{0} \in\{1, \ldots, n\}$ such that $\alpha\left(z_{i_{0}}, P_{i_{0}}\left(x^{*}\right)\right), z_{i_{0}} \in T_{i_{0}}\left(y_{i_{0} j_{0}}, z_{i_{0}}\right)$, which contradicts the fact that $y_{i_{0} j_{0}} \in G_{i_{0}}\left(x^{*}\right)$. Therefore, $x^{*} \notin W_{i}$ for any $i \in I$, which implies that, for each $i \in I, x_{i}^{*} \in S_{i}\left(x^{*}\right)$ and, for any $y_{i} \in Q_{i}\left(x^{*}\right), \alpha\left(z_{i}, P_{i}\left(x^{*}\right)\right), z_{i} \in T_{i}\left(y_{i}, z_{i}\right)$. This completes the proof.

As a generalization of Theorem 3.1, we derive the following existence result for the solution of problem (CFP- $\alpha)$ without convex assumptions.

\section{Theorem 3.7 Assume that}

(i) $X$ is a nonempty and compact subset of a Hausdorff topological vector space $E$, and has the fixed point property, and $Z$ is a Hausdorff topological space;

(ii) $\Omega:=\{x \in X: x \in S(x)\}$ is closed;

(iii) $Q(x) \subset S(x)$ for any $x \in X$;

(iv) $U(y):=\{x \in X \mid \widehat{\alpha}(z, P(x)), z \notin T(y, z)\}$ and $Q^{-1}(y)$ are open in $X$ for any $y \in X$;

(v) for any finite set $\left\{y_{1}, \ldots, y_{n}\right\}$ of $X$, there exists a continuous mapping $\phi_{n}: \Delta_{n} \rightarrow X$ such that

(v1) for any $\lambda=\left(\lambda_{1}, \ldots, \lambda_{n}\right) \in \Delta_{n}$, there exists $i \in J(\lambda)$ such that $\alpha\left(z, P\left(\phi_{n}(\lambda)\right)\right)$,

$$
z \in T\left(y_{i}, z\right) \text {; }
$$

(v2) if $y_{i} \in Q\left(\phi_{n}(\lambda)\right)$ for any $i \in J(\lambda)$, then $\phi_{n}(\lambda) \in Q\left(\phi_{n}(\lambda)\right)$, where

$$
\Delta_{n}:=\left\{\left(\lambda_{1}, \ldots, \lambda_{n}\right) \in \mathbb{R}^{n} \mid \sum_{i=1}^{n} \lambda_{i}=1, \lambda_{i} \geq 0\right\}, \quad J(\lambda):=\left\{i \in\{1, \ldots, n\} \mid \lambda_{i}>0\right\} .
$$


Then problem $(C F P-\alpha)$ has at least a solution, i.e., there exists $\tilde{x} \in X$ such that $\tilde{x} \in S(\tilde{x})$ and, for any $y \in Q(\tilde{x})$,

$$
\alpha(z, P(\tilde{x})), \quad z \in T(y, z) .
$$

Proof Define the mapping $G: X \rightrightarrows X$ as follows:

$$
G(y)=\left[X \backslash Q^{-1}(y)\right] \cup\{x \in X \mid x \in S(x) \text { and } \alpha(z, P(x)), z \in T(y, z)\} .
$$

By (ii) and (iv), $G(y)$ is closed for any $y \in X$.

By (v), for any finite subset $\left\{y_{1}, \ldots, y_{n}\right\}$ of $X$, there exists a continuous mapping $\phi_{n}$ : $\Delta_{n} \longrightarrow X$ such that, for any $\lambda=\left(\lambda_{1}, \ldots, \lambda_{n}\right) \in \Delta_{n}$, there exists $i \in J(\lambda)$ such that $\alpha(z$, $\left.P\left(\phi_{n}(\lambda)\right)\right), z \in T\left(y_{i}, z\right)$, then

(1) if there exists $i_{0} \in J(\lambda)$ such that $y_{i_{0}} \notin Q\left(\phi_{n}(\lambda)\right)$, which implies that $\phi_{n}(\lambda) \in X \backslash Q^{-1}\left(y_{i_{0}}\right)$. Thus $\phi_{n}(\lambda) \in G\left(y_{i_{0}}\right)$;

(2) if $y_{i} \in Q\left(\phi_{n}(\lambda)\right)$ for any $i \in J(\lambda)$, then $\phi_{n}(\lambda) \in Q\left(\phi_{n}(\lambda)\right)$ by (v). By (iii), $\phi_{n}(\lambda) \in S\left(\phi_{n}(\lambda)\right)$. Then $\phi_{n}(\lambda) \in S\left(\phi_{n}(\lambda)\right)$ and, for any $\lambda=\left(\lambda_{1}, \ldots, \lambda_{n}\right) \in \Delta_{n}$, there exists $i \in J(\lambda)$ such that $\alpha\left(z, P\left(\phi_{n}(\lambda)\right)\right), z \in T\left(y_{i}, z\right)$. Thus $\phi_{n}(\lambda) \in G\left(y_{i}\right)$.

Hence, by Theorem 2.1 of [17],

$$
\bigcap_{y \in X} G(y) \neq \emptyset
$$

which implies that there is $\tilde{x} \in X$ such that $\tilde{x} \in S(\tilde{x})$ and, for any $y \in Q(\tilde{x})$,

$$
\alpha(z, P(\tilde{x})), \quad z \in T(y, z) .
$$

By Remarks 3.1 and 3.2, we have the following results.

Theorem 3.8 If (iv) of Theorem 3.7 is replaced by

(a3) $P: X \rightrightarrows Z$ is lower semicontinuous;

(b3) $\{z \in Z \mid z \in T(y, z)\}$ is closed in $X$ for any $y \in Y$, and $Q^{-1}(y)$ is open in $X$ for any $y \in X$. Then problem $\left(C F P-\alpha_{1}\right)$ has at least a solution, i.e., there exists $\tilde{x} \in X$ such that $\tilde{x} \in S(\tilde{x})$ and

$$
z \in \bigcap_{y \in Q(\tilde{x})} T(y, z), \quad \forall z \in P(\tilde{x})
$$

\section{Theorem 3.9 If (iv) of Theorem 3.7 is replaced by}

(a4) $P: X \rightrightarrows Z$ is upper semicontinuous with nonempty compact values;

(b4) $\{z \in Z \mid z \in T(y, z)\}$ is closed in $X$ for any $y \in Y$, and $Q^{-1}(y)$ is open in $X$ for any $y \in X$. Then problem $\left(C F P-\alpha_{2}\right)$ has at least a solution, i.e., there exists $\tilde{x} \in X$ such that $\tilde{x} \in S(\tilde{x})$ and, for any $y \in Q(\tilde{x})$, there is $z \in P(\tilde{x})$ for which $z \in T(y, z)$.

Remark 3.4 In [1-4], convex assumptions or the KKM property played an important role in the proofs of common fixed points. In Theorems 3.7-3.9, the existence of common fixed points does not depend on convex assumptions. 
Remark 3.5 This paper extends the research on common fixed point problems. The classical common fixed point problem (see [1-4]) is a special case of problem (CFP- $\alpha$ ). Moreover, we introduce the system of common fixed points, and common fixed point problems without convex assumptions are obtained.

\section{Applications}

\subsection{Variational inclusions}

In this section, we fix our attention on variational inclusions described below:

Let $X$ be a nonempty, convex and compact subset of a locally convex topological linear space, $Z$ be a Hausdorff topological linear space, and $S, Q: X \rightrightarrows X, A, B: X \times X \rightrightarrows Z$ be set-valued mapping with nonempty values.

A variational inclusion of type $\gamma(\mathrm{VI}-\gamma)$ consists in finding $\tilde{x} \in X$ such that $\tilde{x} \in S(\tilde{x})$ and $\gamma(A(\tilde{x}, y), B(\tilde{x}, y))$ holds for any $y \in Q(\tilde{x})$.

A variational inclusion of type $\gamma_{1}\left(\mathrm{VI}-\gamma_{1}\right)$ consists in finding $\tilde{x} \in X$ such that $\tilde{x} \in S(\tilde{x})$ and $A(\tilde{x}, y) \subset B(\tilde{x}, y)$ for any $y \in Q(\tilde{x})$.

A variational inclusion of type $\gamma_{2}\left(\mathrm{VI}-\gamma_{2}\right)$ consists in finding $\tilde{x} \in X$ such that $\tilde{x} \in S(\tilde{x})$ and $A(\tilde{x}, y) \cap B(\tilde{x}, y) \neq \emptyset$ holds for any $y \in Q(\tilde{x})$.

Theorem 4.1 Let $X$ be a nonempty, convex and compact subset of a locally convex topological linear space, $Z$ be a Hausdorff topological linear space. Assume that

(i) $S$ is upper semicontinuous with nonempty convex compact values;

(ii) $Q^{-1}(y)$ is open in $X$ for any $y \in X$, and $\emptyset \neq Q(x) \subset S(x)$ for any $x \in X$;

(iii) the set $\{x \in X \mid \gamma(A(x, y), B(x, y))$ holds $\}$ is closed in $X$ for any $y \in X$;

(iv) for any finite set $\left\{x_{1}, \ldots, x_{n}\right\}$ of $X$ and any $x \in \operatorname{co}\left\{x_{1}, \ldots, x_{n}\right\}$, there is $i_{0} \in\{1, \ldots, n\}$ such that $\gamma\left(A\left(x, x_{i_{0}}\right), B\left(x, x_{i_{0}}\right)\right)$ holds.

Then problem $(V I-\gamma)$ has at least a solution.

Proof Define the mapping $T: X \times X \rightrightarrows X$ as follows:

$$
T(y, x)=\{z \in X: \gamma(A(x, y), B(z, y)) \text { holds }\} .
$$

By (iii), $\{x \in X: x \in T(y, x)\}$ is closed in $X$ for any $y \in X$. By Corollary 3.1, there exists $\tilde{x} \in$ $X$ such that $\tilde{x} \in S(\tilde{x})$ and $\tilde{x} \in \bigcap_{y \in Q(\tilde{x})} T(y, \tilde{x})$, which implies $\tilde{x} \in S(\tilde{x})$ and $\gamma(A(\tilde{x}, y), B(\tilde{x}, y))$ holds for any $y \in Q(\tilde{x})$. This completes the proof.

Theorem 4.2 Assume (ii) and (iii) of Theorem 4.1 are replaced by

(a5) for any $y \in X, A(\cdot, y)$ is lower semicontinuous, and $B(\cdot, y)$ is closed;

(b5) for any finite set $\left\{x_{1}, \ldots, x_{n}\right\}$ of $X$ and any $x \in \operatorname{co}\left\{x_{1}, \ldots, x_{n}\right\}$, there is $i_{0} \in\{1, \ldots, n\}$ such that $A\left(x, x_{i_{0}}\right) \subset B\left(x, x_{i_{0}}\right)$.

Then problem $\left(V I-\gamma_{1}\right)$ has at least a solution.

Proof As soon as we show that the set $\{x \in X: A(x, y) \subset B(x, y)\}$ is closed for any $y \in X$. Let $\left\{x^{\alpha}\right\}$ be a net in $X$ converging to $x$, such that $A\left(x^{\alpha}, y\right) \subset B\left(x^{\alpha}, y\right)$ for any $\alpha$. For any $z \in A(x, y)$, since $A(\cdot, y)$ is lower semicontinuous, by Lemma 2.2 , there is $z^{\alpha} \in A\left(x^{\alpha}, y\right) \subset B\left(x^{\alpha}, y\right)$ such that $z^{\alpha} \longrightarrow z$. It follows from the closeness of $B(\cdot, y)$ that $z \in B(x, y)$. Then $A(x, y) \subset B(x, y)$. Thus, the set $\{x \in X: A(x, y) \subset B(x, y)\}$ is closed for any $y \in X$. 
Theorem 4.3 Assume (ii) and (iii) of Theorem 4.1 are replaced by

(a6) for any $y \in X, A(\cdot, y)$ is upper semicontinuous with nonempty compact values, and $B(\cdot, y)$ is closed;

(b6) for any finite set $\left\{x_{1}, \ldots, x_{n}\right\}$ of $X$ and any $x \in \operatorname{co}\left\{x_{1}, \ldots, x_{n}\right\}$, there is $i_{0} \in\{1, \ldots, n\}$ such that $A\left(x, x_{i_{0}}\right) \cap B\left(x, x_{i_{0}}\right) \neq \emptyset$.

Then problem $\left(V I-\gamma_{2}\right)$ has at least a solution.

Proof As soon as we show that the set $\{x \in X: A(x, y) \cap B(x, y) \neq \emptyset\}$ is closed for any $y \in X$. Let $\left\{x^{\alpha}\right\}$ be a net in $X$ converging to $x$, such that $A\left(x^{\alpha}, y\right) \cap B\left(x^{\alpha}, y\right) \neq \emptyset$ for any $\alpha$. Then there exists $z^{\alpha} \in Z$ such that $z^{\alpha} \in A\left(x^{\alpha}, y\right) \cap B\left(x^{\alpha}, y\right)$ for any $\alpha$. Since $A(\cdot, y)$ is upper semicontinuous with nonempty compact values, by (iii) of Lemma 2.2 , there is a subnet $\left\{z^{\alpha \beta}\right\}$ of $\left\{z^{\alpha}\right\}$ converging to some $z \in A(x)$. Since $z^{\alpha \beta} \in B\left(x^{\alpha \beta}, y\right)$, and $B(\cdot, y)$ is closed, $z \in B(x, y)$. Thus $z \in A(x, y) \cap B(x, y)$. Hence, the set $\{x \in X: A(x, y) \cap B(x, y) \neq \emptyset\}$ is closed for any $y \in X$.

\subsection{Generalized multiplied minimax inequality of Ky Fan type}

Let $X$ be a nonempty, convex and compact subset of a locally convex topological linear space, and $S, Q: X \rightrightarrows X, f: X \times X \times X \rightarrow \mathbb{R}$ be a real-valued function. A generalized multiplied minimax inequality of Ky Fan type consists in finding $\tilde{x} \in X$ such that $\tilde{x} \in S(\tilde{x})$ and $f(y, \tilde{x}, \tilde{x}) \leq 0$ for any $y \in Q(\tilde{x})$.

Theorem 4.4 Let $X$ be a nonempty, convex and compact subset of a locally convex topological linear space. Assume that

(i) $S$ is upper semicontinuous with nonempty convex compact values;

(ii) $Q^{-1}(y)$ is open in $X$ for any $y \in X$, and $\emptyset \neq Q(x) \subset S(x)$ for any $x \in X$;

(iii) $f(y, \cdot, \cdot)$ is lower semicontinuous on $X \times X$ for any $y \in X$;

(iv) for any finite set $\left\{x_{1}, \ldots, x_{n}\right\}$ of $X$ and any $x \in \operatorname{co}\left\{x_{1}, \ldots, x_{n}\right\}$, there is $i_{0} \in\{1, \ldots, n\}$ such that $f\left(x_{i_{0}}, x, x\right) \leq 0$.

Then the generalized multiplied minimax inequality of Ky Fan type has at least a solution.

Proof Define the mapping $T: X \times X \rightrightarrows X$ as follows:

$$
T(y, x)=\{z \in X: f(y, x, z) \leq 0\} .
$$

By (iii), $\{x \in X: x \in T(y, x)\}$ is closed in $X$ for any $y \in X$. By Corollary 3.1, there exists $\tilde{x} \in X$ such that $\tilde{x} \in S(\tilde{x})$ and $\tilde{x} \in \bigcap_{y \in Q(\tilde{x})} T(y, \tilde{x})$, which implies $\tilde{x} \in S(\tilde{x})$ and $f(y, \tilde{x}, \tilde{x}) \leq 0$ for any $y \in Q(\tilde{x})$. This completes the proof.

Remark 4.1 Theorem 4.4 is different from Theorems 4.2, 4.3 of [4]. (1) Our Theorem 4.4 with constraining mappings $S, Q$ is a more general problem than Theorems 4.2, 4.3 of [4]. (2) The existence conditions are different between Theorem 4.4 and Theorems 4.2, 4.3 of [4].

From Theorem 4.4, when $S(x)=Q(x)=X$ for any $x \in X$, we obtain a multiplied minimax inequality of Ky Fan type.

Theorem 4.5 Let $X$ be a nonempty, convex and compact subset of a locally convex topological linear space. Assume that 
(i) $f(y, \cdot, \cdot)$ is lower semicontinuous on $X \times X$ for any $y \in X$;

(ii) for any finite set $\left\{x_{1}, \ldots, x_{n}\right\}$ of $X$ and any $x \in \operatorname{co}\left\{x_{1}, \ldots, x_{n}\right\}$, there $i s i_{0} \in\{1, \ldots, n\}$ such that $f\left(x_{i_{0}}, x, x\right) \leq 0$.

Then the multiplied minimax inequality of Ky Fan type has at least a solution, i.e., $f(y, \tilde{x}, \tilde{x}) \leq 0$ for any $y \in X$.

\section{Competing interests}

The author declares that they have no competing interests.

\section{Acknowledgements}

This research is supported by the Chen Guang Project sponsored by the Shanghai Municipal Education Commission and Shanghai Education Development Foundation (no. 13CG35), and open project of Key Laboratory of Mathematical Economics (SUFE), Ministry of Education (no. 201309KF02).

Received: 21 April 2014 Accepted: 5 August 2014 Published: 02 Sep 2014

\section{References}

1. Balaj, M: A common fixed point theorem with applications to vector equilibrium problems. Appl. Math. Lett. 23 241-245 (2010)

2. Agarwal, RP, Balaj, M, O'Regan, D: Common fixed point theorems and minimax inequalities in locally convex Hausdorff topological vector spaces. Appl. Anal. 88, 1691-1699 (2009)

3. Lin, LJ, Chuang, CS, Yu, ZT: Generalized KKM theorems and common fixed point theorems. Nonlinear Anal. 74 5591-5599 (2011)

4. Agarwal, RP, Balaj, M, O'Regan, D: A common fixed point theorem with applications. J. Optim. Theory Appl. (2013). doi:10.1007/s10957-013-0490-6

5. Luc, DT: An abstract problem in variational analysis. J. Optim. Theory Appl. 138, 65-76 (2008)

6. Khanh, PQ, Luc, DT: Stability of solutions in parametric variational relation problems. Set-Valued Anal. 16, 1015-1035 (2008)

7. Pu, YJ, Yang, Z: Stability of solutions for variational relation problems with applications. Nonlinear Anal. 75, 1758-1767 (2012)

8. Lin, LJ, Wang, SY: Simultaneous variational relation problems and related applications. Comput. Math. Appl. 58, 1711-1721 (2009)

9. Balaj, M, Luc, DT: On mixed variational relation problems. Comput. Math. Appl. 60, 2712-2722 (2010)

10. Balaj, M, Lin, LJ: Equivalent forms of a generalized KKM theorem and their applications. Nonlinear Anal. 73, 673-682 (2010)

11. Balaj, M, Lin, LJ: Generalized variational relation problems with applications. J. Optim. Theory Appl. 148, 1-13 (2011)

12. Lin, LJ, Ansari, QH: Systems of quasi-variational relations with applications. Nonlinear Anal. 72, 1210-1220 (2010)

13. Agarwal, RP, Balaj, M, O'Regan, D: A unifying approach to variational relation problems. J. Optim. Theory Appl. 155 417-429 (2012)

14. Balaj, M, Lin, LJ: Existence criteria for the solutions of two types of variational relation problems. J. Optim. Theory Appl. $156,232-246(2013)$

15. Luc, DT, Sarabi, E, Soubeyran, A: Existence of solutions in variational relation problems without convexity. J. Math. Anal. Appl. 364, 544-555 (2010)

16. Pu, YJ, Yang, Z: Variational relation problem without the KKM property with applications. J. Math. Anal. Appl. 393, 256-264 (2012)

17. Yang, Z, Pu, YJ: Generalized Knaster-Kuratowski-Mazurkiewicz theorem without convex hull. J. Optim. Theory Appl. $154,17-29(2012)$

18. Yang, Z, Pu, YJ: Existence and stability of solutions for maximal element theorem on Hadamard manifolds with applications. Nonlinear Anal. 75, 516-525 (2012)

19. Yang, Z: On existence and essential stability of solutions of symmetric variational relation problems. J. Inequal. Appl. 2014, 5 (2014)

20. Hung, NV: Sensitivity analysis for generalized quasi-variational relation problems in locally G-convex spaces. Fixed Point Theory Appl. 2012, 158 (2012)

21. Hung, NV: Continuity of solutions for parametric generalized quasi-variational relation problems. Fixed Point Theory Appl. 2012, 102 (2012)

22. Latif, A, Luc, DT: Variational relation problems: existence of solutions and fixed points of contraction mappings. Fixed Point Theory Appl. 2013, 315 (2013)

23. Aliprantis, CD, Border, KC: Infinite Dimensional Analysis: A Hitchhiker's Guide, 3rd edn. Springer, Berlin (2006)

24. Deguire, $P$, Tan, KK, Yuan, GXZ: The study of maximal elements, fixed points for $L_{s}$-majorized mapping and their applications to minimax and variational inequalities in the product topological spaces. Nonlinear Anal. 37, 933-951 (1999)

10.1186/1687-1812-2014-189

Cite this article as: Yang: Some generalizations of common fixed point problems with applications. Fixed Point Theory and Applications 2014, 2014:189 